\title{
Control de Tensión en el Bus DC para Compensadores Activos de Potencia Conectados en Paralelo
}

\author{
Leonardo E. Torres-Acevedo, Jorge H. Urrea-Quintero, Nicolás Muñoz-Galeano \\ Fac. de Ingeniería, Depto Ing. Eléctrica, Grupo de Manejo Eficiente de la Energía - GIMEL, Universidad de \\ Antioquia, Calle 67 No. 53-108, Oficina 19-437, Medellín, Colombia \\ (e-mail: leonardo.torres@udea.edu.co, humberto.urrea@udea.edu.co,nicolas.munoz@udea.edu.co)
}

Recibido Mar. 30, 2017; Aceptado May. 30, 2017; Versión final Jul. 14, 2017, Publicado Dic. 2017

\begin{abstract}
Resumen
Este artículo aborda el problema del control de tensión en el bus DC para compensadores activos de potencia conectados en paralelo (SAPC, Shunt Active Power Compensator) de tres ramas y cuatro hilos, basando la solución en su modelo matemático. A partir del modelo del SAPC se establece un modelo con información suficiente para deducir la función de transferencia que describe el proceso de carga y descarga del bus DC. Se emplea la sintonía de un controlador clásico PI con la velocidad y robustez suficientes para mantener el nivel de tensión adecuado en el bus DC, de tal modo que, la red solo suministra la potencia activa que la carga demanda. El modelo propuesto garantiza la compensación de potencia reactiva, la regulación de la tensión en el bus DC, y el equilibrio entre los dos capacitores del enlace del bus DC. Los resultados se verifican a partir del modelo matemático en MATLAB y se validan por medio de un modelo circuital implementado en PSIM.
\end{abstract}

Palabras clave: electrónica de potencia; Bus DC; control; compensador activo de potencia, SAPC

\section{Voltage Control of a DC Bus for Shunt Active Power Compensators}

\begin{abstract}
This paper deals with the DC-bus control problem for three-leg four-wires Shunt Active Power Compensators (SAPCs), based on the solution of a mathematical model. Starting with the SAPC concept a mathematical model with enough information to deduce the transfer function that describes the charging/discharging DC-bus process is proposed. A PI controller for tuning the SAPC with enough speed and robustness to keep the DC voltage in an adequate level is employed, so that it is guaranteed that the active power is only supplied on demand. The proposed model guarantees reactive power compensation, regulation of the DC bus, and balance between the two capacitors in the DC bus. The mathematical model is verified by Matlab simulations and it is validated through a circuital model implemented in PSIM.
\end{abstract}




\section{INTRODUCCIÓN}

Los sistemas de potencia son susceptibles a problemas de calidad de la potencia debido a la conexión de cargas reactivas, desequilibradas y no lineales. Para mitigar dichos problemas, se plantea como solución el uso de SAPCs. Sin embargo, para que un SAPC pueda funcionar correctamente es necesario mantener la tensión en el bus DC controlada, garantizando que se mantenga alrededor del valor nominal (Orts et al., 2010). Para el control del bus DC, los investigadores o ingenieros desarrolladores utilizan generalmente controladores del tipo PI (Proporcional e Integral) o PID (Proporcional, Integral y Derivativo) que tienden a ser cada vez más complejos. Sriranjani y Jayalalitha (2011) sintonizan controladores PI y PID para el control del bus DC. El controlador PI permitió obtener una respuesta rápida, pero con sobrepico en la tensión del bus DC, mientras que con el controlador PID el sistema se vuelve más lento, pero mejora la respuesta del sobrepico de tensión. Los autores no indican la forma cómo se obtienen los parámetros de los controladores. Otros autores (Lam et al., 2012; Wang et al., 2011) implementan un control adaptable PI para el bus DC, el cual tiene la capacidad de cambiar la tensión de referencia del bus DC de acuerdo a las condiciones de carga a las que esté sometido. No obstante, el rango de operación del bus DC es determinado por las especificaciones de diseño. Wang et al. (2015) implementan un controlador PID modificado con seguimiento diferenciador (TD, Tracking-Differentiator), con el objetivo de mejorar el desempeño dinámico del bus DC, logrando disminuir el sobre-pico y el tiempo de establecimiento. Sin embargo, no detalla la sintonía del controlador e incluye parámetros sin llevar a cabo el desarrollo para obtenerlos.

Guo et al. (2012) proponen la implementación de un controlador PI difuso para controlar el bus DC, los autores utilizan dos controladores difusos que actúan sobre las ganancias proporcional e integral. Aunque el control PI difuso reduce el sobre-pico y disminuye el error en estado estable, su implementación requirió un alto costo computacional. (Liu et al., 2010; Dazhi et al., 2010) proponen la sintonía de controladores PI/PID difusos para múltiples puntos de operación; de tal forma que, mejoran el error en estado estable, mejoran el desempeño dinámico y proporcionan mayor robustez. Sin embargo, la implementación y el diseño de este tipo de controladores son complejos, requiriendo desde el diseño un mayor esfuerzo. Rodriguez et al. (2008) proponen controladores que calculan las referencias de corrientes con base en los estados de energía del SAPC para dimensionar los capacitores del bus DC de acuerdo a la respuesta dinámica del sistema. No obstante, en estos estudios no se incluyen la velocidad de respuesta del controlador y a los sobre picos de tensión en el bus DC. Choi et al. (2013) proponen el control del bus DC usando componentes de corriente en eje directo y de cuadratura, ellos enfatizan en la carga inicial del bus DC e indican que mejoran la respuesta dinámica del sistema. Villalobos y Suárez (2010) proponen un algoritmo para controlar individualmente cada uno de los capacitores de un SAPC TLSC (Three-Leg Split Capacitor); sin embargo, cuando el SAPC requiere compensar componentes de corriente de secuencia cero, el algoritmo no mantiene el balance en cada uno de los capacitores.

La revisión de la literatura muestra que para controlar mejor el bus DC, los investigadores recurren a implementar controladores cada vez más complejos. El modelo propuesto incluye las pérdidas (resistencias) en las bobinas y condensadores para una aproximación más realista, esto permitió prescindir de controladores complejos pudiéndose utilizar controladores clásicos PI. Se resalta de nuestra investigación que se realizó un esfuerzo mayor en el modelado del sistema, pero es posible desde la etapa de diseño saber las limitaciones o restricciones del sistema. El modelo propuesto también permite presentar un procedimiento para: el diseño y sintonía de los controladores $\mathrm{PI}$, de tal forma que se garantiza la compensación y equilibrio del bus DC mientras se realiza la inyección de potencia reactiva a la red.

\section{MODELADO DEL SAPC TRIFASICO}

En la Fig. 1 se ilustra el SAPC trifásico considerado en nuestra investigación, incluye un inversor de 3 fases a 4 hilos y de capacitor dividido (TLSC, Three-Leg Split-Capacitor) (Khadkikar et al., 2011), se seleccionó esta topología por las prestaciones que entrega con un menor número de interruptores de potencia. Cada una de las 3 ramas de las fases $(A, B, C)$ del SAPC está compuesta por dos interruptores de potencia y se conectan en el punto de conexión común en la carga. El bus DC está compuesto por dos capacitores y de su punto medio se obtiene el neutro $(N)$ del sistema, en este punto se conecta el neutro de la red trifásica y de la carga. Como interfaz de conexión entre el SAPC y la red trifásica se dispone de tres bobinas que sirven como filtros de armónicos y como elemento de conexión entre el inversor y red trifásica. Para obtener una aproximación más realista del sistema se incluyen las resistencias de conducción de las bobinas de acople $\left(R_{L a}=R_{L b}=\right.$ $\left.R_{L c}=R_{L}\right)$, y las resistencias de pérdidas en los capacitores $\left(R_{C 1}=R_{C 2}=R_{C}\right)$. Del SAPC mostrado en la Fig. 1 y usando las leyes circuitales de Kirchhoff se obtiene el modelo en coordenadas abc; el cual se representa mediante las ecuaciones diferenciales (1), (2) y (3). Donde $i_{L}^{a b c}$ es la corriente del inductor en las fases $a, b$ y $c$; $v_{d c}$ es la tensión del bus DC $\left(v_{d c}=v_{c 1}-v_{c 2}\right) ; u^{a b c}$ es la función de conmutación para las fases $a, b$ y $c$ $\left(u^{a b c} \in \mathcal{R}^{3 x 1}\right.$, donde para el caso de los interruptores superiores abiertos y los interruptores de las inferiores cerrados, de las fase $a, b$ y $c, u^{a b c}$ toma valores positivos en el intervalo $[0,1]$ y para el caso de los interruptores 
superiores cerrados y los interruptores inferiores abiertes $u^{a b c}$ toma valores negativos en el intervalo $\left.[-1,0]\right)$; $\epsilon_{v}$ permite verificar el desequilibrio de voltaje en el bus $\mathrm{DC}\left(\epsilon_{v}=\left(v_{c 1}+v_{c 2}\right) / 2\right)$; y $v_{p c c}^{a b c}$ es la tensión en el punto de conexión común (pcc) donde se conectan la carga, la red y el SAPC.

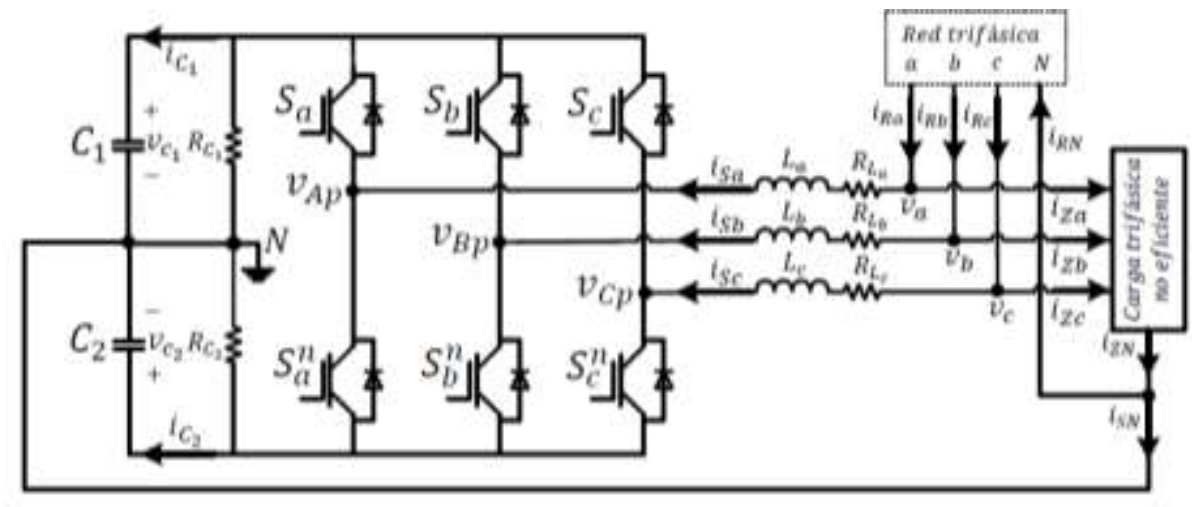

Fig. 1: Topología del SAPC trifásico

$L \frac{d i_{L}^{a b c}}{d t}=-R_{L} i_{L}^{a b c}-\frac{v_{d c}}{2} u^{a b c}-\epsilon_{v}+v_{p c c}^{a b c}$

$C \frac{d v_{d c}}{d t}=i_{L}^{a b c} u^{a b c^{T}}-\frac{v_{d c}}{R_{c}}$

$2 C \frac{d \epsilon_{v}}{d t}=i_{L}^{a b c}-2 \frac{\epsilon_{v}}{R_{C}}$

El análisis dinámico y el diseño del control son más complejos para sistemas que varían en el tiempo que para aquellos sistemas invariantes en el tiempo, por lo que se propone el cambio a coordenadas dq0 (Sun et al., 2009). La transformada $d q 0$ permite cambiar el sistema de referencia en coordenadas rectangulares $a b c$ por un sistema de coordenadas rotatorio $d q 0$ el cual gira a la velocidad de la frecuencia de la red, logrando que la señal sinusoidal de frecuencia fundamental se vea reflejada como una señal DC. Una ventaja de la transformada $d q 0$ es que para sistemas sin desbalance; la componente $d$ está directamente relacionada con la potencia activa, mientras que la componente $q$ está relacionada a la potencia reactiva (Reginatto y Ramos, 2014). La ecuación (4) corresponde a la matriz de transformación $T$ que se utiliza para llevar un sistema de referencia $a b c$ a $d q 0$, donde $\omega$ corresponde a la frecuencia de la red eléctrica y $\theta$ al ángulo de sincronización de la transformada.

$$
T=\frac{2}{3}\left[\begin{array}{ccc}
\sin (\omega t+\theta) & \sin \left(\omega t-\frac{2 \pi}{3}+\theta\right) & \sin \left(\omega t+\frac{2 \pi}{3}+\theta\right) \\
\cos (\omega t+\theta) & \cos \left(\omega t-\frac{2 \pi}{3}+\theta\right) & \cos \left(\omega t+\frac{2 \pi}{3}+\theta\right) \\
\frac{1}{2} & \frac{1}{2} & \frac{1}{2}
\end{array}\right]
$$

Dos propiedades útiles de la transformada $d q 0$ son enunciadas en las ecuaciones (5) y (6)

$$
\begin{aligned}
& {[T]^{-1}=[T]^{T}} \\
& {[T] \frac{d}{d t}[T]^{T}=\omega\left[\begin{array}{ccc}
0 & -1 & 0 \\
1 & 0 & 0 \\
0 & 0 & 0
\end{array}\right]}
\end{aligned}
$$

Aplicando la transformada $d q 0$ (4) y sus propiedades (5) y (6) al modelo del SAPC en coordenadas abc (1), (2) y (3), se obtiene el modelo del SAPC en $d q 0$ (7), (8) y (9). El modelo dq0 permitirá el uso de controladores simples del tipo PI para alcanzar fácilmente el estado estable cuando se perturba el sistema.

$$
L\left[\left[\begin{array}{c}
-\omega i_{L}^{q} \\
\omega i_{L}^{d} \\
0
\end{array}\right]+\frac{d i_{L}^{d q 0}}{d t}\right]=-R_{L} i_{L}^{d q 0}-\frac{v_{d c}}{2} u^{d q 0}-\left[\begin{array}{c}
0 \\
0 \\
\sqrt{3}
\end{array}\right] \epsilon_{v}+v_{p c c}^{d q 0}
$$




$$
\begin{aligned}
& C \frac{d v_{d c}}{d t}=i_{L}^{d q 0} u^{d q 0^{T}}-\frac{v_{d c}}{R_{C}} \\
& 2 C \frac{d \epsilon_{v}}{d t}=\sqrt{3} i_{L}^{0}-2 \frac{\epsilon_{v}}{R_{C}}
\end{aligned}
$$

\section{ESTRUCTURA DE CONTROL}

Para el control del bus DC se considera una estructura de control en cascada, con ella se obtiene mayor estabilidad en la tensión del bus DC (Xu et al., 2014). En la Fig. 2.a) se muestra el esquema simplificado de la estructura utilizada, internamente se tiene un lazo de control de corriente y en la parte exterior se tiene un controlador de tensión. La señal de entrada al controlador externo es la tensión de referencia del bus DC la cual es comparada con la tensión actual del bus DC, dicha diferencia ingresa a un controlador PI. La señal de salida del controlador PI externo se compara con la corriente de eje directo en la salida del SAPC, posteriormente el error de corriente es llevado al controlador PI interno o controlador de corriente. La señal de salida de este controlador es la señal moduladora de eje directo que modifica la corriente de eje directo y a su vez la tensión en el bus DC.

(a)

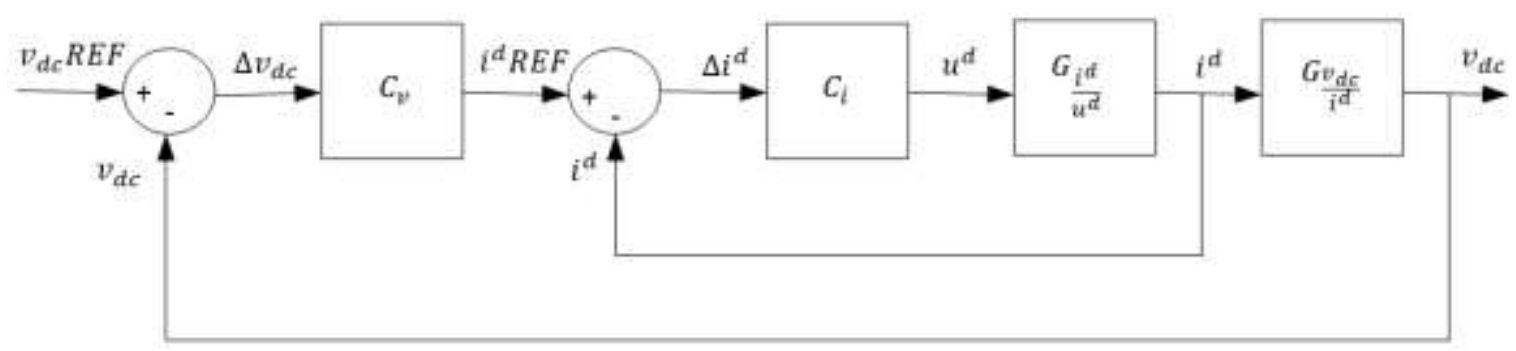

(b)

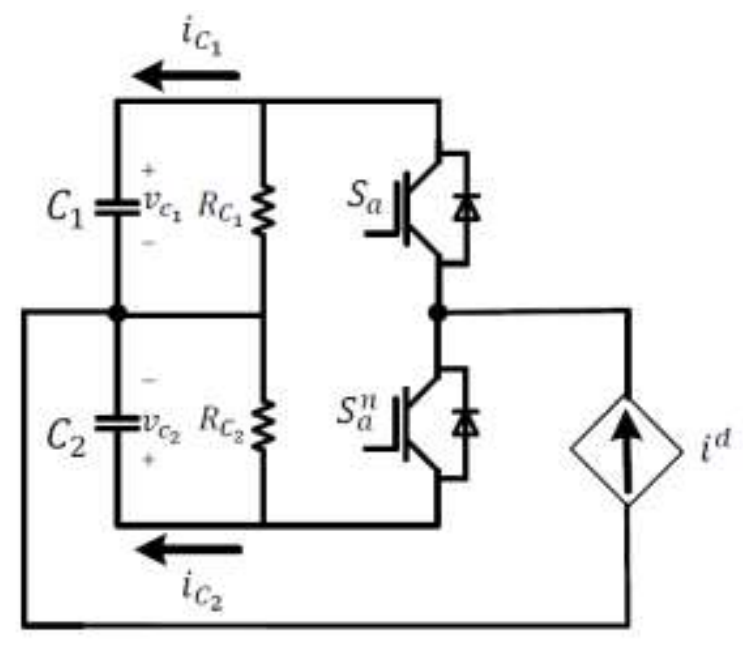

Fig. 2: a) Estructura de control del bus DC. b) Circuito equivalente para el modelo simplificado del bus DC

Si se considera que el lazo interno se encuentra bien sintonizado, de tal manera que su respuesta dinámica sea suficientemente rápida respecto a la respuesta dinámica del bus DC, dicho lazo puede ser simplificado mediante una función de transferencia que incluye una fuente de corriente controlada conectada al bus DC (Fig. 2.b). Del circuito de la Fig. 2.b) se deduce la ecuación dinámica del bus DC, la cual está dada por (10). De (10) se puede deducir la función de transferencia para el diseño del controlador de voltaje o controlador externo de la estructura de control en cascada.

$$
C \frac{d v_{d c}}{d t}=i^{d} u^{d}-\frac{v_{d c}}{R_{C}}
$$

\section{DEDUCCION DE LA FUNCION DE TRANSFERENCIA DEL BUS DC}

EI SAPC debe suministrar potencia reactiva a la carga y a su vez cargar el bus DC desde la red, bajo estas premisas, el SAPC inyecta y/o requiere componentes de corriente balanceada. En condiciones balanceadas las componentes de secuencia cero son cero; es decir, los siguientes términos de las ecuaciones (7), (8) y (9) 
son cero: $v_{p c c}^{0}, i_{L}^{0}, u^{0}, \frac{d i_{L}^{0}}{d t} y \frac{d \epsilon_{v}}{d t}$. Para el sistema en condiciones balanceadas, se tiene la representación en espacio de estados ilustrada en las ecuaciones (11) y (12).

$$
\begin{aligned}
& {\left[\begin{array}{c}
i_{L}^{d} \\
\dot{\iota_{L}^{q}} \\
v_{d c}
\end{array}\right]=\underbrace{\left[\begin{array}{ccc}
\frac{-R_{L}}{L} & \omega & \frac{-u^{d}}{2 L} \\
\omega & \frac{-R_{L}}{L} & \frac{-u^{q}}{2 L} \\
\frac{u^{d}}{C} & \frac{u^{q}}{C} & \frac{-1}{R_{C} C}
\end{array}\right]}_{A}\left[\begin{array}{c}
i_{L}^{d} \\
i_{L}^{q} \\
v_{d c}^{q}
\end{array}\right]+\underbrace{\left[\begin{array}{cc}
\frac{-v_{d c}}{2 L} & 0 \\
0 & \frac{-v_{d c}}{2 L} \\
\frac{i_{L}^{d}}{C} & \frac{i_{L}^{q}}{C}
\end{array}\right]}_{B}\left[\begin{array}{c}
u^{d} \\
u^{q}
\end{array}\right]} \\
& y=\underbrace{\left[\begin{array}{lll}
1 & 0 & 0 \\
0 & 1 & 0 \\
0 & 0 & 1
\end{array}\right]}_{C}\left[\begin{array}{c}
i_{L}^{d} \\
i_{L}^{q} \\
v_{d c}^{q}
\end{array}\right]+\underbrace{\left[\begin{array}{lll}
0 & 0 & 0 \\
0 & 0 & 0
\end{array}\right]}_{D}\left[\begin{array}{c}
u^{d} \\
u^{q}
\end{array}\right]
\end{aligned}
$$

De las ecuaciones (11) y (12) se obtienen directamente las funciones de transferencia del sistema al aplicar la transformada de Laplace (Goodwin et al, 2001). Para la relación entrada salida $i_{L}^{d} / u^{d}$, se obtiene la función de transferencia dada por la ecuación (13).

$$
G(s)=\left[\begin{array}{ll}
\frac{i_{L}^{d}}{u^{d}} & \frac{i_{L}^{d}}{u^{q}} \\
\frac{i_{L}^{q}}{u^{d}} & \frac{i_{L}^{q}}{u^{q}} \\
\frac{v_{d c}}{u^{d}} & \frac{v_{d c}}{u^{q}}
\end{array}\right]
$$

De (13) se toma $G_{i_{L}^{d} / u^{d}}=\frac{i_{L}^{d}}{u^{d}}(14)$, esta función de transferencia sirve para sintonizar el controlador de corriente.

$$
\begin{aligned}
& G_{i_{L}^{d} / u^{d}}= \\
& \frac{s^{2}\left(\frac{-v_{d c}}{2 L}\right)+s\left(-\frac{L v_{d c}+C R_{C} R_{L} v_{d c}+L R_{C} i_{L}^{d} u^{d}}{2 C L^{2} R_{C}}\right)+\left(-\frac{L R_{C} v_{d c} u^{q^{2}}+2 L R_{C} i_{L}^{d} \omega u^{q}+2 R_{L} v_{d c}+2 R_{C} R_{L} i_{L}^{d} u^{d}}{4 C L^{2} R_{C}}\right)}{s^{3}+s^{2}\left(\frac{L+2 C R_{C} R_{L}}{C L R_{C}}\right)+s\left(\frac{2 C R_{C} L^{2} \omega^{2}+4 L R_{L}+R_{C} L u^{d^{2}}+R_{C} L u^{q^{2}}+2 C R_{C} R_{L}^{2}}{2 C L^{2} R_{C}}\right)+\left(\frac{2 L^{2} \omega^{2}+2 R_{L}^{2}+R_{C} R_{L} u^{d^{2}}+R_{C} R_{L} u^{q^{2}}}{2 C L^{2} R_{C}}\right)}
\end{aligned}
$$

El controlador externo es sintonizado con base en la función de transferencia deducida de la ecuación dinámica simplificada del bus DC (10), y corresponde a la función de transferencia $G_{v_{d c} / i_{L}^{d}}=\frac{v_{d c}}{i_{d}}$, (15).

$$
G_{v_{d c} / i_{L}^{d}}=\frac{R_{C} u^{d}}{R_{C} C s+1}
$$

\section{RESULTADOS}

Para la validación del modelo y de las funciones de transferencia, se realizaron simulaciones del SAPC de la Fig. 1, para esto se eligieron como valores nominales: 1) voltaje en la red de $120 V_{R M S}$, 2) frecuencia del voltaje en la red de $50 \mathrm{~Hz}, 3$ ) voltaje nominal del bus DC de $600 V_{D C}$, 4) potencia aparente nominal de la carga de $1869 \mathrm{VA}, 5)$ potencia activa nominal de la carga $1500 \mathrm{~W}, 6)$ potencia reactiva nominal de la carga $1115 \mathrm{Var}$, 7) factor de potencia nominal de la carga de 0,8 Inductivo y 8) frecuencia de conmutación del inversor de $20 \mathrm{kHz}$. En cuanto a los parámetros del sistema se eligieron: 1) capacitores del bus DC de $2200 \mu F, 2)$ resistencia de pérdidas en el bus DC $1 k \Omega$, 3) inductancia de acople de $30.2 \mathrm{mH}, 4$ ) resistencia de pérdidas de las inductancias de acople de $1 \Omega$.

Con el objetivo de mostrar que el modelo matemático representa el sistema implementado en el software PSIM, se realizaron simulaciones en el dominio de la frecuencia, esta validación se muestra en la Fig. 3, en esta se observa que ambos modelos siguen la misma tendencia desde los $10 \mathrm{~Hz}$ hasta $1 \mathrm{MHz}$, este ajuste garantiza que dentro del rango de frecuencia de operación el modelo matemático representa adecuadamente el modelo circuital. 

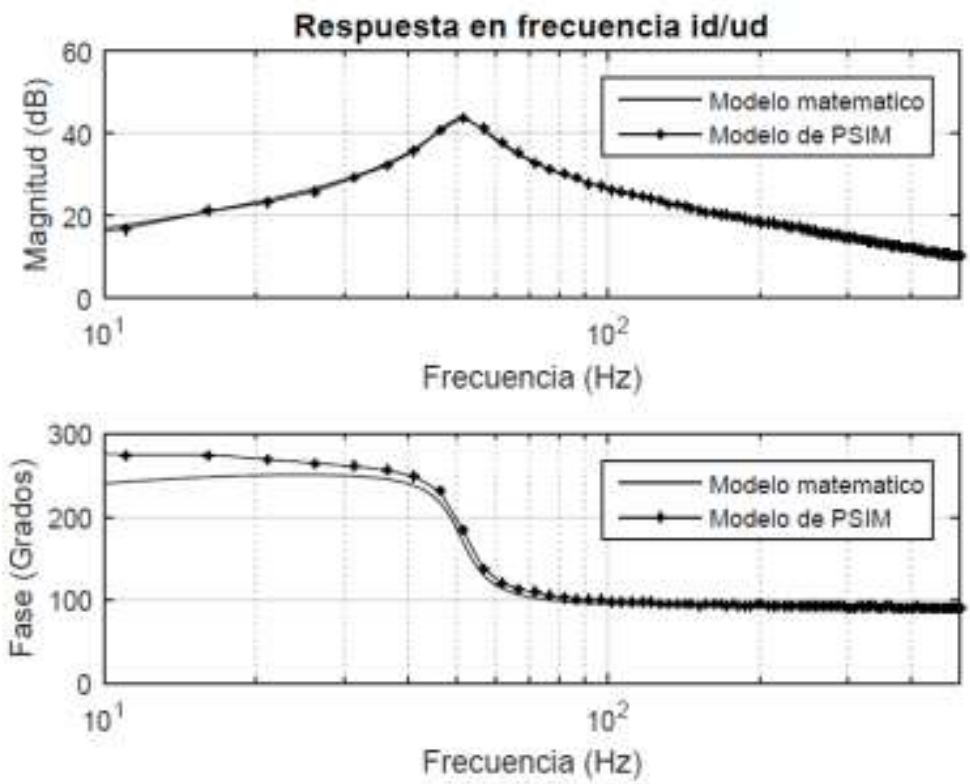

Fig. 3: Respuesta en frecuencia para $G_{i_{L}^{d} / u^{d}}$

La sintonía de los controladores se realizó usando la herramienta SISOTOOL del toolbox de control de MATLAB, los controladores se sintonizaron con un factor de amortiguamiento y ancho de banda que garantizaran un compromiso entre la robustez y la velocidad del controlador (Vilanova, 2011), para ambos controladores se estableció un factor de amortiguamiento de 0,707 , un ancho de banda de $25 \mathrm{~Hz}$ para el controlador de tensión y un ancho de banda de $4 \mathrm{kHz}$ el controlador de corriente, el cual corresponde a una quinta parte la frecuencia de conmutación (Louganski y Lai., 207). La ganancia proporcional y el tiempo integral de los controladores para el lazo de corriente son $\left(K_{p}=1.8054\right.$ y $\left.T_{i}=0.00012 \mathrm{~s}\right)$, mientras que para el lazo de tensión son $\left(K_{p}=0.336262\right.$ y $\left.T_{i}=0.019000 \mathrm{~s}\right)$. Los diagramas de bode del sistema en lazo cerrado se muestran en la Fig. 4.a) para la corriente y en la Fig. 4.b) para el voltaje, en ambos diagramas se verifica que la frecuencia de corte corresponda a la de diseño del controlador, esto es, $4 \mathrm{kHz}$ para el lazo de corriente y $25 \mathrm{~Hz}$ para el lazo de voltaje. En ambos diagramas también se verifica que el sistema es estable en lazo cerrado pues el margen de ganancia y el margen de fase se encuentran dentro de la zona de estabilidad. Además, se observa que el controlador garantiza un seguimiento perfecto de la referencia para señales con frecuencias por debajo de la frecuencia de corte y un error en estado estacionario igual a cero.
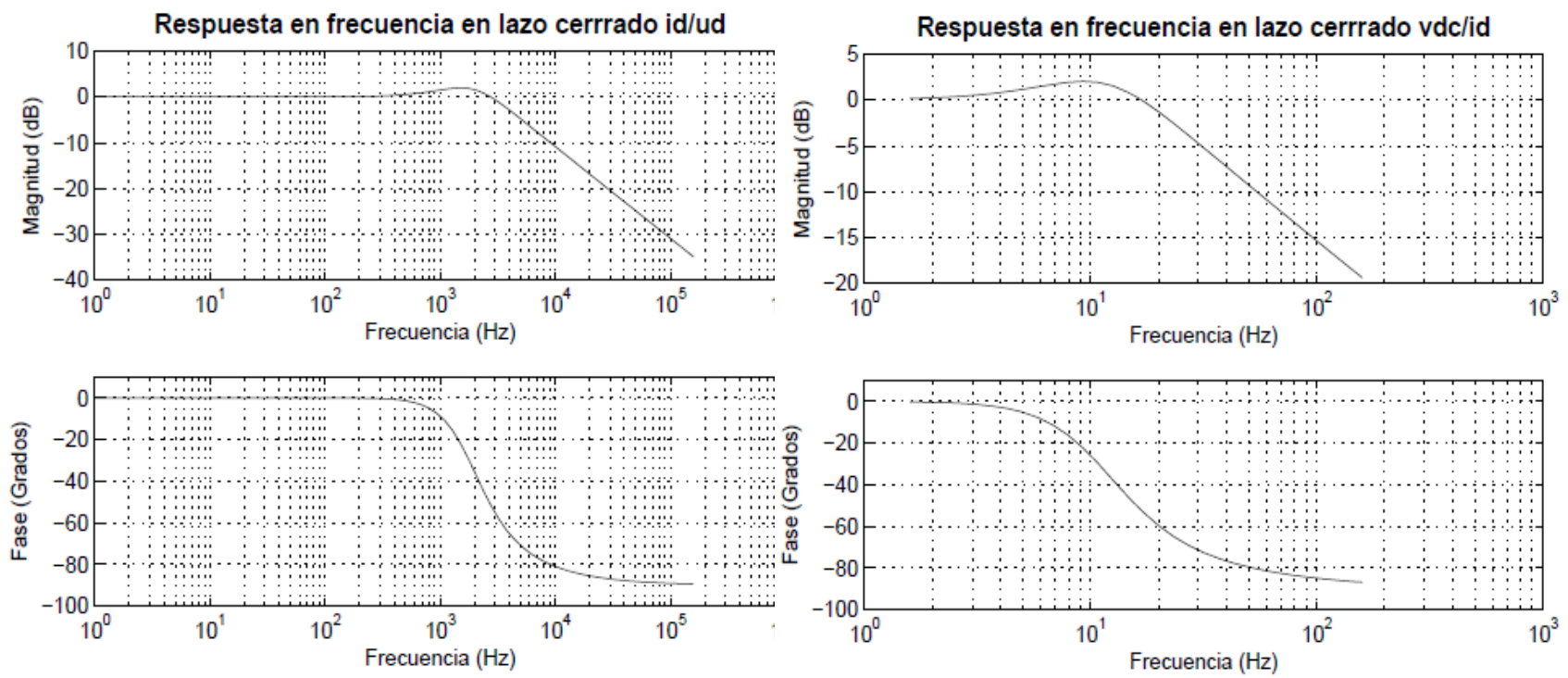

Fig. 4: Respuesta en frecuencia de lazo cerrado para a) $G_{i_{L}^{d} / u^{d}}$ b) $G_{v_{d c} / i_{L}^{d}}$

En la Fig. 5 se muestra la respuesta dinámica del SAPC en lazo cerrado ante múltiples cambios en el valor nominal de la carga. Para probar el desempeño de los controladores PI fueron aplicados tres cambios del tipo escalón en el valor nominal de la corriente de carga así: entre los 0 y 1 segundos el sistema opera con el valor nominal de la carga, a los 1 segundos el valor nominal de carga se reduce al $95 \%$, a los 1.5 segundos el valor 
nominal de la carga se reduce al $90 \%$, a los 2 segundos el valor nominal de la carga se aumenta hasta el $110 \%$ de su valor nominal. Siendo este último el cambio más agresivo para los controladores. La Fig. 5.a) muestra el comportamiento del voltaje (línea sólida) y el valor de referencia para el controlador de voltaje (línea punteada) en el bus DC. De la Fig. 5.a) se puede observar que ante los diferentes cambios en el valor nominal de la carga el controlador es capaz de reestablecer el valor nominal del voltaje en el bus DC. Esto fue logrado con transitorios del orden de los 0.1 segundos y con valores de sobrepico en la respuesta menores al $0.2 \%$. La Fig. 5.b) muestra el comportamiento de la componente directa de la corriente (línea punteada) y su referencia (línea sólida) a la salida del SAPC. De la Fig. 5.b) es posible observar que la componente directa de la corriente a la salida del SAPC sigue satisfactoriamente su referencia. La Fig. 5.c) muestra el comportamiento de la componente en cuadratura de la corriente (línea punteada) y su referencia (línea sólida) a la salida del SAPC. De la Fig. 5.c) es posible observar que la componente en cuadratura de la corriente sigue satisfactoriamente su referencia. La Fig. 5.d) muestra el comportamiento de las componentes directa y en cuadratura para la señal moduladora que recibe el SAPC. En términos generales, de la Fig. 5 es posible concluir que los controladores de corriente cumplen bien su función a pesar de que aparecen algunos sobrepicos en los transitorios.

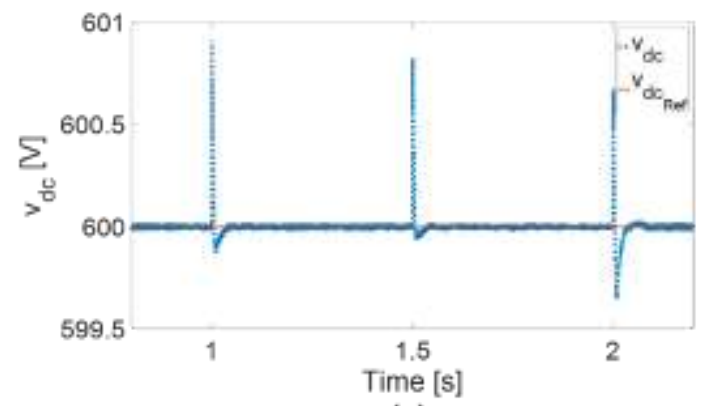

(a)

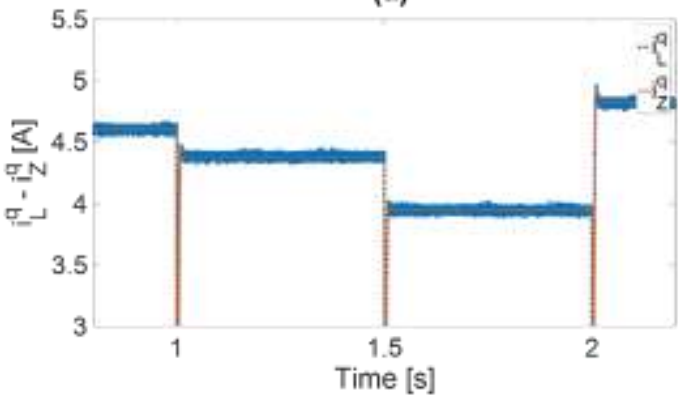

(c)

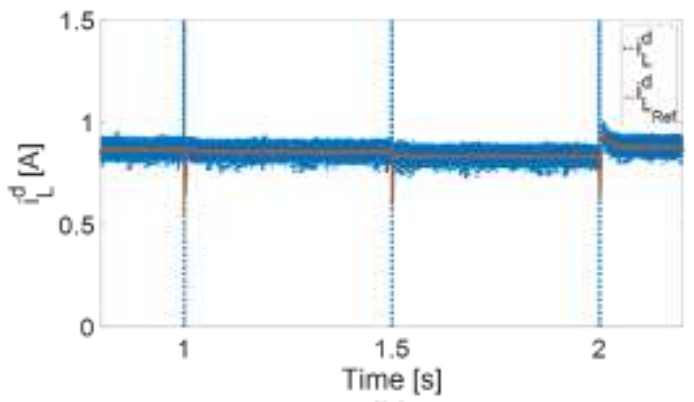

(b)

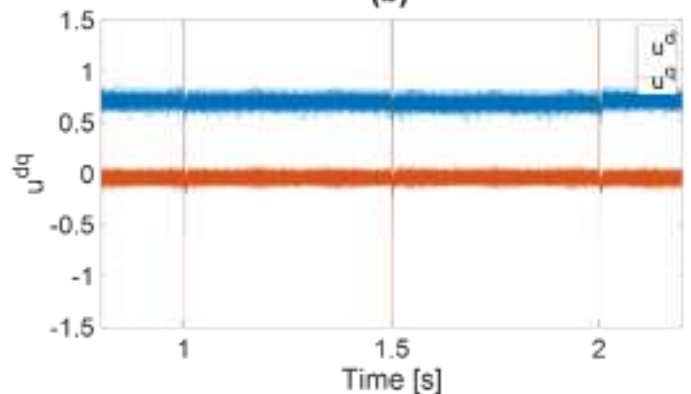

(d)

Fig. 5: Respuesta dinámica del SAPC en lazo cerrado: (a) voltaje en el bus DC, (b) componente directa de la corriente a la salida del SAPC, (c) componente en cuadratura de la corriente a la salida del SAPC, (d) componente directo y en cuadratura de la señal moduladora.

En la Fig. 6 se muestran los voltajes y las corrientes que la fuente de suministro entrega a la carga. Las Fig. 6.a), 6.b) y 6.c) representan las fases A, B y C, respectivamente. En la Fig. 6 se puede observar que las tres corrientes están en fase con sus respectivos voltajes. Las Fig. Las Fig. 6.a), 6.b) y 6.c) contienen un zoom en el intervalo $1.5-1.65$ segundos, allí se puede apreciar que a pesar de que a los 1.5 segundos es aplicada una perturbación al sistema, los voltajes y las corrientes se vuelven a poner en fase apenas transcurrido un instante de tiempo de aproximadamente 5 ciclos de la señal.

En la Fig. 7 se muestran las señales de las corrientes para las componentes directa y en cuadratura que entregan la red de suministro, que se generan a la salida del SAPC y que demanda la carga. En la Fig. 7.a) se muestra la componente directa de la corriente para la fuente de suministro, el SAPC y la carga. De la Fig. 7.a) es posible observar que la fuente de suministro entrega toda la corriente relacionada con la potencia activa que el sistema demanda, es decir, suple simultáneamente la corriente de componente directa que la carga demanda y que el SAPC requiere para mantener el voltaje en el bus DC en su valor nominal. Lo anterior se comprueba restándole a la corriente de la fuente de suministro la corriente que demanda el SAPC. Como se puede ver en la Fig. 7.a), al graficar la corriente de componente directa que demanda la carga (línea negra sólida) y la diferencia entre la corriente en componente directa de la fuente de suministro y el SAPC (línea punteada), estas dos coinciden. La Fig. 7.b) muestra las corrientes de componente en cuadratura que entrega la red de suministro, la que se genera a la salida del SAPC y la que demanda la carga. De la Fig. 7.b) es posible observar que para la red de suministro, el valor de la componente en cuadratura de la corriente es cero para todo el tiempo de simulación. Esto implica que la red de suministro solo le entrega potencia activa al sistema. Por otro lado, de la Fig. 7.b) es posible observar que la corriente para la componente en cuadratura entregada por el SAPC coincide con la demanda por la carga; por lo tanto, el SAPC es el que suple la potencia reactiva que la carga demanda. 


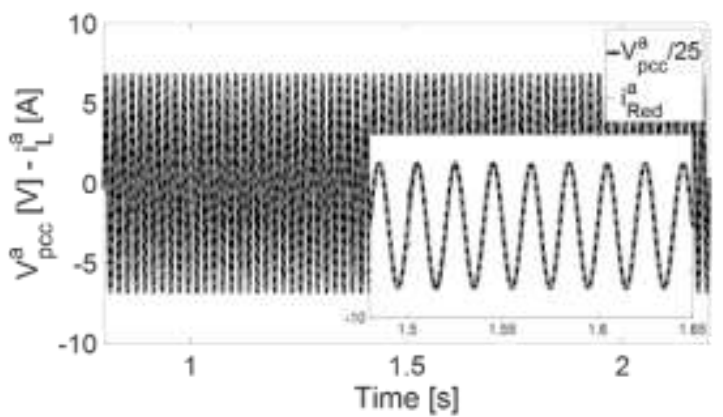

(a)

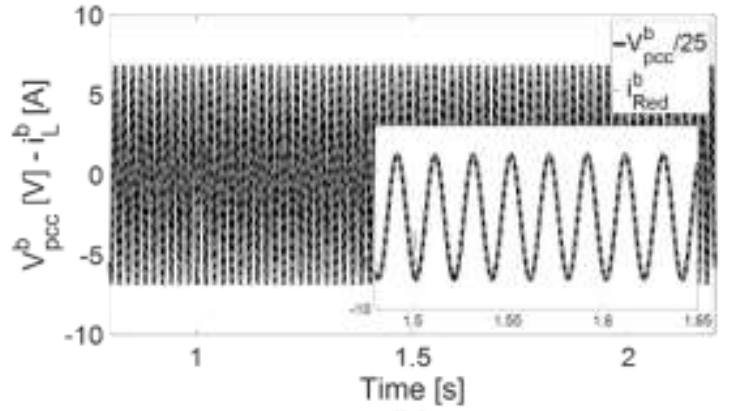

(b)

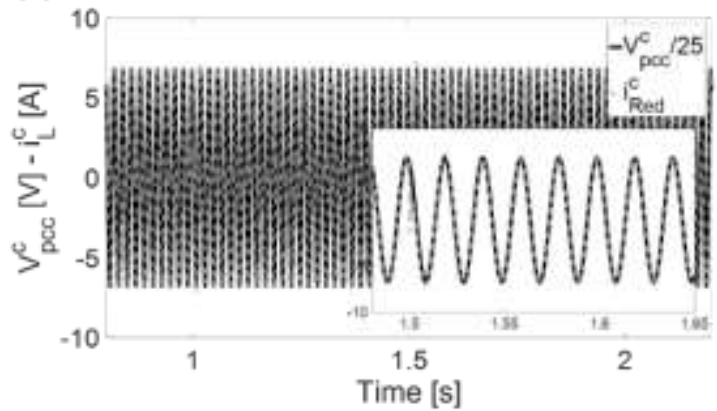

(c)

Fig. 6: Voltajes y corrientes suministrados por la fuente: (a) fase A, (b) fase B, (c) fase C.

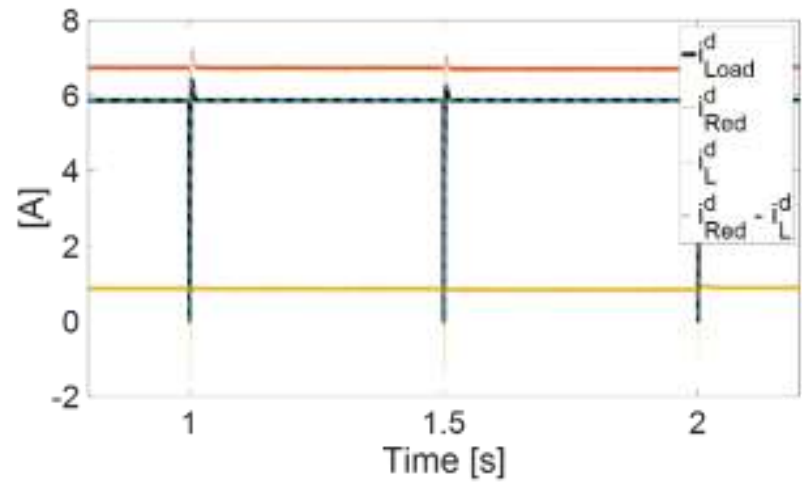

(a)

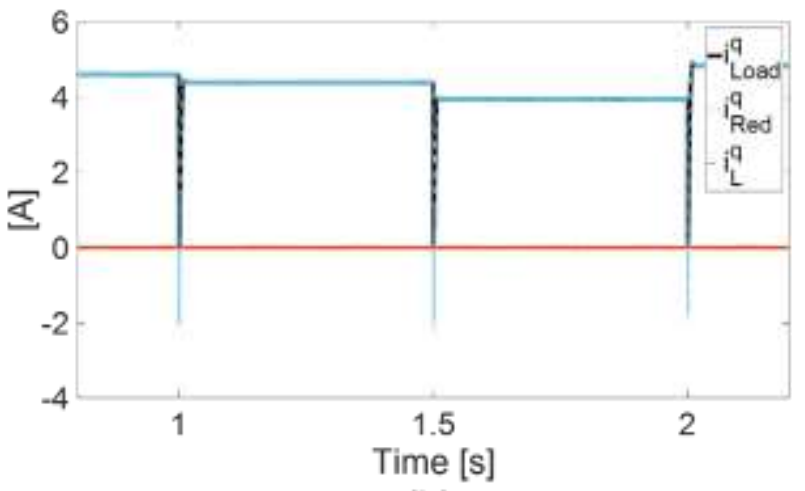

(b)

Fig. 7: Corrientes directas y en cuadratura en el sistema: (a) componente directa, (b) componente en cuadratura

\section{CONCLUSIONES}

En el artículo se planteó el modelo matemático para el SAPC que incluye las pérdidas de los elementos pasivos. La respuesta en frecuencia del modelo matemático se comparó con la respuesta en frecuencia del sistema simulado en PSIM, se encontró que ambas respuestas se ajustan bastante bien y siguen la misma tendencia desde los $10 \mathrm{~Hz}$ hasta $1 \mathrm{MHz}$, aunque existe una pequeña diferencia en los diagramas de fase en el rango de frecuencia de $10 \mathrm{~Hz}$ a $100 \mathrm{~Hz}$. Esta diferencia se debe a que el modelo matemático corresponde con el modelo lineal del SAPC, mientras que la simulación en PSIM que corresponde a su modelo no lineal; en este caso, la linealización del sistema indujo algo de incertidumbre, sin embargo la estrategia de control que se planteó, que es una estrategia de control simple del tipo PI, tolera dicho error de modelado.

Se resalta el hecho de con el modelo propuesto fue posible construir una estructura de control que permitió al SAPC satisfacer simultáneamente múltiples objetivos de control, de tal modo que, fue posible suplir las potencias reactiva demandada por la carga al mismo tiempo que se mantuvo el nivel de tensión en el bus DC, ambas cosas sin afectar el desempeño global del sistema ni su estabilidad. Adicional a esto para mantener el sistema operando, solo se requirió de la red de suministro potencia activa, lo que hace del sistema un sistema de alta eficiencia energética.

El modelo propuesto permito el uso de controladores en cascada PI, con un control de corriente en el lazo interno y un control de tensión en el lazo externo. Los resultados mostraron que la estrategia de control, ante cambios en el valor nominal de la corriente de carga es capaz de reestablecer el valor nominal del voltaje en el bus DC, logrando transitorios del orden de los 0.1 segundos y con valores de sobrepico en la respuesta menores al $0.2 \%$. 
Se resalta que la componente en cuadratura de la corriente que permite el control de la potencia reactiva sigue satisfactoriamente su referencia. En cuanto a la tensión de suministro, se mostró que al aplicar una perturbación al sistema, los voltajes y las corrientes se vuelven a poner en fase en un tiempo aproximado de 5 ciclos de la señal. Bajos las condiciones de operación reflejadas en las Fig. 5, 6 y 7 es posible concluir que el sistema operando en lazo cerrado con el SAPC cumple satisfactoriamente con su objetivo y que el modelo propuesto para el diseño del controlador de tensión es consistente con los resultados esperados.

\section{AGRADECIMIENTOS}

Los autores agradecen a la Universidad de Antioquia (UdeA) por el apoyo del programa de sostenibilidad y al proyecto codi 2015-7747.

\section{REFERENCIAS}

Choi, W.H., C.S. Lam, M.C. Wong y Y.D. Han. Analysis of DC-Link Voltage Controls in Three-Phase FourWire Hybrid Active Power Filters. IEEE Transactions on Power Electronics, 28(5), 2180-2191 (2013)

Goodwin, G.C., S. F. Graebe y M. E. Salgado. Control system design. Prentice Hall, Upper Saddle River, USA (2001) Guo, X., D. Wang y R. Chen. Active Power Filter DC Bus Voltage Control Based on Fuzzy PI Compound Control. World Automation Congress, 1-4 (2012)

Khadkikar, V., A. Chandra y B. Singh. Digital Signal Processor Implementation and Performance Evaluation of Split Capacitor, Four-Leg and Three H-Bridge-Based Three-Phase Four-Wire Shunt Active Filters. IET Power Electronics, 4(4), 463-70 (2011)

Lam, C. S., W. H. Choi, M. C. Wong y Y. D. Han. Adaptive DC-Link Voltage-Controlled Hybrid Active Power Filters for Reactive Power Compensation. IEEE Transactions on Power Electronics, 27(4), 1758-1772 (2012)

Liu, Z., T. Ma, S. Ge y B. Wang. Hybrid Active Power Filter DC Bus Control Based on Fuzzy PID Control. International Symposium on Computational Intelligence and Design, 2, 75-78 (2010)

Louganski, K. P. y J. S. Lai. Current Phase Lead Compensation in Single-Phase PFC Boost Converters With a Reduced Switching Frequency to Line Frequency Ratio. IEEE Transactions on Power Electronics, 22(1), 113-119 (2007)

Orts, S., A. Abellán, F. Gimeno, S. Seguí, y J. Alfonso. Improved Shunt Active Power Compensator for IEEE Standard 1459 Compliance, IEEE Trans. Power Delivery, 25 (4), 2692-2701 (2010)

Reginatto, R. y R. A. Ramos. On Electrical Power Evaluation in Dq Coordinates under Sinusoidal Unbalanced Conditions. IET Generation Transmission \& Distribution, 8(5), 976-982 (2014)

Rodriguez, P., A. Luna, R. Teodorescu, F. Blaabjerg y M. Liserre. Control of a Three-Phase Four-Wire ShuntActive Power Filter Based on DC-Bus Energy Regulation. 11th International Conference on Optimization of Electrical and Electronic Equipment, 227-234 (2008)

Sriranjani, R. y S. Jayalalitha. Improvement of the Time Domain Specifications of Dc Bus Voltage of Shunt Active Filter Using Controllers. International Conference on Recent Advacements in Electrical, Electronics and Control Engineering, 187-191 (2011)

Sun, Z., Z. Zhang y T. Tsao. Trajectory Tracking and Disturbance Rejection for Linear Time-Varying Systems: Input/output Representation. Systems \& Control Letters, 58(6), 452-460 (2009)

Vilanova, R. y V.M. Alfaro. Control PID Robusto: Una Visión Panorámica. Revista lberoamericana de Automática e Informática Industrial RIAI, 8(3), 141-158 (2011)

Villalobos, M., A. Mantilla y J.F. Petit. DC Bus Voltage Control in Four-Wire Shunt Active Power Filters with Split Capacitor. IEEE/PES Transmission and Distribution Conference and Exposition: Latin America (T\&D-LA), 751-758 (2010)

Wang, C., X. Zong y X. Cheng. Control DC Bus Voltage of Active Power Filter with a Novel PID Control. IEEE International Conference on Information and Automation, 1132-137 (2015)

Wang, D., S. Ge, J. Li, B. Wang, K. Song, X. Liu y Y. Li. Hybrid Active Power Filter DC Bus Control Based on Double Fuzzy Control. 2nd Inter. Conference on Computational Intelligence and Natural Computing, 1, 287-290 (2010)

Wang, D., X. Wang, K. Song, Y. Li y S. Liu. An Adaptive Control Method of Shunt Active Power Filter DC Bus Voltage. Second International Conference on Digital Manufacturing \& Automation, 172-176 (2011)

Xu, Q., X. Zhong, W. Yao y G. Chen. High Precision Control Strategy for Three-Phase Four-Wire Shunt Active Power Filter. IEEE 23rd International Symposium on Industrial Electronics (ISIE), 509-514 (2014) 
